\title{
Chapter 1 \\ Nuclear Transmutation of Long-Lived \\ Nuclides with Laser Compton Scattering: \\ Quantitative Analysis by Theoretical Approach
}

\author{
Shizuka Takai and Kouichi Hagino
}

\begin{abstract}
A photo-neutron $(\gamma, \mathrm{n})$ reaction with laser Compton scattering $\gamma$-rays has been suggested to be effective for the nuclear transmutations of fission products. The photo-neutron reaction occurs via a giant dipole resonance, which has a large cross section and whose properties are smooth functions of mass number. The laser Compton scattering can generate effectively and selectively high-energy photons with a desired energy range. In this chapter, we investigate quantitatively the effectiveness of the transmutation with laser Compton scattering based on the Hauser-Feshbach theory using the TALYS code. We carry out simulations for high-decay heating nuclide ${ }^{137} \mathrm{Cs}$, in which the cross sections for ${ }^{137} \mathrm{Cs}(\gamma, \gamma),(\gamma, \mathrm{n})$, and $(\gamma, 2 n)$ reactions, and the total photonuclear reaction cross sections versus incident photon energy, are calculated. The incident photon energy obtained by laser Compton scattering is also optimized. It is shown that the transmutation with medium-energy photon with a flux of more than $10^{18} / \mathrm{s}$ effectively reduces the radioactivity of the target ${ }^{137} \mathrm{Cs}$.
\end{abstract}

Keywords ${ }^{137} \mathrm{Cs} \cdot$ Giant dipole resonance $\cdot$ Laser Compton scattering • Photoneutron reaction $\bullet$ Radioactive wastes $\bullet$ Transmutation

\subsection{Introduction}

One of the major problems of the nuclear fuel cycle is the disposal of high-level radioactive waste that contains long-lived nuclides such as ${ }^{129} \mathrm{I}$ and high-decay heating nuclides such as ${ }^{137} \mathrm{Cs}$. After the severe accident at the Fukushima Daiichi

\footnotetext{
S. Takai $(\bowtie)$

Nuclear Safety Research Center, Japan Atomic Energy Agency, Tokai-mura, Naka-gun, Ibaraki 319-1115, Japan

e-mail: takai.shizuka@jaea.go.jp

K. Hagino

Department of Physics, Tohoku University, Sendai, Miyagi 980-8578, Japan 
Nuclear Power Plant, there is also a problem of ${ }^{137} \mathrm{Cs}$ having been concentrated by treatment of contaminated water. Transmuting such nuclides into short-lived or stable nuclides is one possible way to resolve this problem. Neutron capture reactions have been proposed for transmutations of such fission products. However, the neutron capture cross sections differ significantly from nuclide to nuclide, and this transmutation method is not effective for nuclides with small neutron capture cross sections such as ${ }^{137} \mathrm{Cs}$.

Recently, photo-neutron $(\gamma, n)$ reactions with laser Compton scattering $\gamma$-rays have been suggested as an alternative method for nuclear transmutations $[1,2]$. Figure 1.1 shows a schematic illustration of this transmutation. This transmutation uses $\gamma$-rays generated by laser photons backscattered off $\mathrm{GeV}$ electrons and photonuclear reactions via electric giant dipole resonance (GDR) [3], which has a large cross section for most nuclides. The GDR is a collective excitation of a nucleus that decays mainly by the emission of neutrons, and its total cross section is a smooth function of mass number. Therefore, this method is expected to be effective for transmuting fission products regardless of isotopes.

So far, transmutation with laser Compton scattering for some nuclides has been evaluated only in a simple manner. In this chapter, we investigate more quantitatively the effectiveness of the transmutation with laser Compton scattering, especially for ${ }^{137} \mathrm{Cs}$.

\subsection{Calculation Method}

\subsubsection{Reaction via Giant Dipole Resonance}

Nuclear transmutation with laser Compton scattering uses photonuclear reactions via GDR because the cross section of GDR is quite large and the total cross section is a smooth function of mass number. GDR is a collective excitation of a nucleus involving almost all nucleons, which is interpreted classically as a macroscopic oscillation of a bulk of protons against that of neutrons. The total cross section $\sigma_{\mathrm{GDR}}^{\mathrm{tot}}$, the resonance energy $E_{R}$, and the width $\Gamma_{R}$ are given by [4]

$$
\begin{gathered}
\sigma_{\mathrm{GDR}}^{\mathrm{tot}}=60(1+\kappa) \frac{N Z}{A} \mathrm{mb} \cdot \mathrm{MeV} \\
E_{R}=31.2 \mathrm{~A}^{-1 / 3}+20.6 \mathrm{~A}^{-1 / 6} \mathrm{MeV} \\
\Gamma_{R}=0.0026 E_{R}^{1.91} \mathrm{MeV}
\end{gathered}
$$

where $N$ and $Z$ are the neutron and proton numbers, $A=N+Z$ is the mass number, and $\kappa$, which is roughly equal to 0.2 for medium nuclides, is a correction coefficient for the pion exchange.

When a target nucleus is irradiated with a photon and excited to GDR, it often forms a compound nucleus with only a small contribution of a pre-equilibrium reaction [5]. The compound nucleus is an excited state in which the energy brought 
Fig. 1.1 Schematic illustration of nuclear transmutation with laser Compton scattering
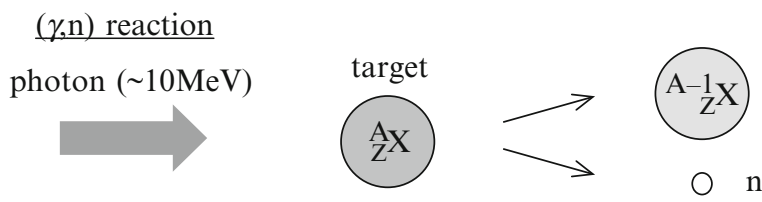

Giant Dipole Resonance : GDR (collective excitation)

by the incident particle is shared among all degrees of freedom of the nucleus. The reaction cross section from an initial channel $\alpha$ to a final channel $\beta$ proceeding through a compound nucleus state of spin $J$ can be written by the Hauser-Feshbach formula as

$$
\sigma_{\alpha \beta}^{c n}=\frac{\pi}{k_{\alpha}^{2}} \sum_{J} g_{J} \frac{T_{\alpha}\left\langle T_{\beta}\right\rangle}{\sum_{\gamma}\left\langle T_{\gamma}\right\rangle},
$$

where $k_{\alpha}$ is the wave number in the initial channel, $g_{J}$ is a statistical factor, $T$ is a transmission coefficient, and $\langle T\rangle$ is the energy average of $T$. The statistical factor is

$$
g_{J}=\frac{2 J+1}{\left(2 i_{\alpha}+1\right)\left(2 I_{\alpha}+1\right)},
$$

where $i_{\alpha}$ and $I_{\alpha}$ are the projectile and target spins.

Calculations of reaction cross sections are performed using the nuclear model code TALYS (version 1.4) [6]. The neutron transmission coefficients are calculated via the global optical potential [7]. The gamma-ray transmission coefficients are calculated through the energy-dependent gamma-ray strength function according to Brink [8] and Axel [9]. We employed the level density given by Gilbert and Cameron [10].

Figure 1.2 shows the photonuclear reaction cross sections of ${ }^{137} \mathrm{Cs}$ calculated using the TALYS code. In the incident photon energy $B(\mathrm{n}) \leq E_{\gamma}<B(2 \mathrm{n})$, where $B$ (n) and $B(2 \mathrm{n})$ are the one- and two-neutron binding energies, respectively, we can see that the $(\gamma, \mathrm{n})$ reaction mainly occurs. Because the resonance energy of GDR $E_{R}$ is $15-18 \mathrm{MeV}$, which is roughly equal to $B(2 \mathrm{n})$ for medium nuclides, about half the reactions via GDR are $(\gamma, \mathrm{n})$ reactions, which occur at $B(\mathrm{n}) \leq E_{\gamma}<B(2 \mathrm{n})$.

\subsubsection{High-Energy Photons Obtained by Laser Compton Scattering}

Laser Compton scattering is a method to obtain high-energy photons by laser photons backscattered off energetic $\mathrm{GeV}$ electrons. In the case of head-on collision 


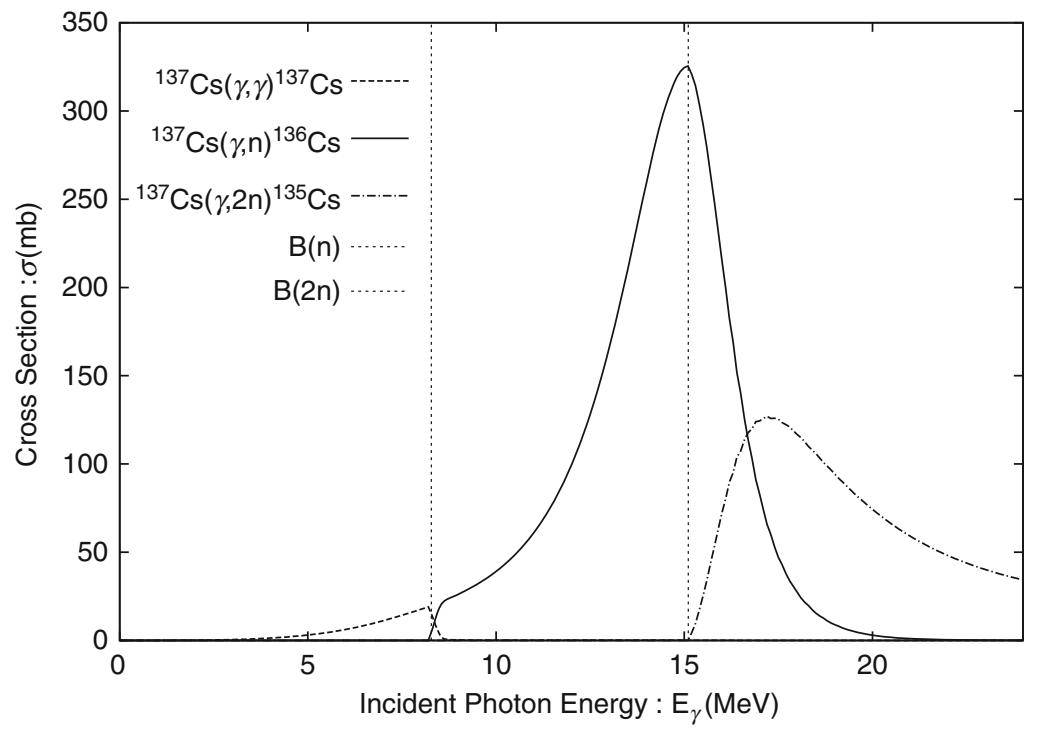

Fig. 1.2 Cross sections for ${ }^{137} \mathrm{Cs}(\gamma, \gamma),{ }^{137} \mathrm{Cs}$ (dashed line), ${ }^{137} \mathrm{Cs}(\gamma, \mathrm{n}),{ }^{136} \mathrm{Cs}$ (solid line), and ${ }^{137} \mathrm{Cs}(\gamma, 2 \mathrm{n}) .{ }^{135} \mathrm{Cs}$ (dash-dot line) reactions versus incident photon energy: dotted lines represent $B(\mathrm{n})$ and $B(2 \mathrm{n})$ of ${ }^{137} \mathrm{Cs}$

between relativistic electrons and laser photons, the energy of scattered photons is given by

$$
E_{\gamma}=\frac{4 \gamma^{2} E_{L}}{1+(\gamma \theta)^{2}+4 E_{L} \gamma / m_{e}},
$$

where $\gamma=E_{e} / m_{e}$ is the Lorentz factor of the electron beam with energy $E_{e}, m_{e}$ is the rest mass of the electron, $E_{L}$ is the energy of the laser photon, and $\theta$ is the scattering angle. From Eq. (1.4), the energy of the scattered photon is maximum at $\theta=0$, and it depends on the energy of incident electrons and photons. The minimum energy of the scattered photon can be fixed by controlling $\theta$ with collimators.

The scattering cross section of laser Compton scattering is given by the KleinNishina formula:

$$
\begin{gathered}
\frac{\mathrm{d} \sigma}{\mathrm{d} E_{\gamma}}=\frac{\pi r_{0}^{2}}{2} m_{e}^{2} E_{L} E_{e}^{2}\left\{\frac{m_{e}^{4}}{4 E_{L}^{2} E_{e}^{2}}\left(\frac{E_{e}}{E_{\gamma}-E_{e}}\right)^{2}-\frac{m_{e}^{2}}{4 E_{L} E_{e}}\left(\frac{E_{e}}{E_{\gamma}-E_{e}}\right)+\frac{E_{e}}{E_{\gamma}-E_{e}}+\frac{E_{\gamma}-E_{e}}{E_{e}}\right\}, \\
N_{\gamma}=\int \mathrm{d} E_{\gamma} \frac{r_{0}=e^{2} / 4 \pi m_{e}}{\mathrm{~d} E_{\gamma}}=\int \mathrm{d} E_{\gamma} \frac{\mathrm{d} \sigma}{\mathrm{d} E_{\gamma}} \cdot \text { const. }
\end{gathered}
$$




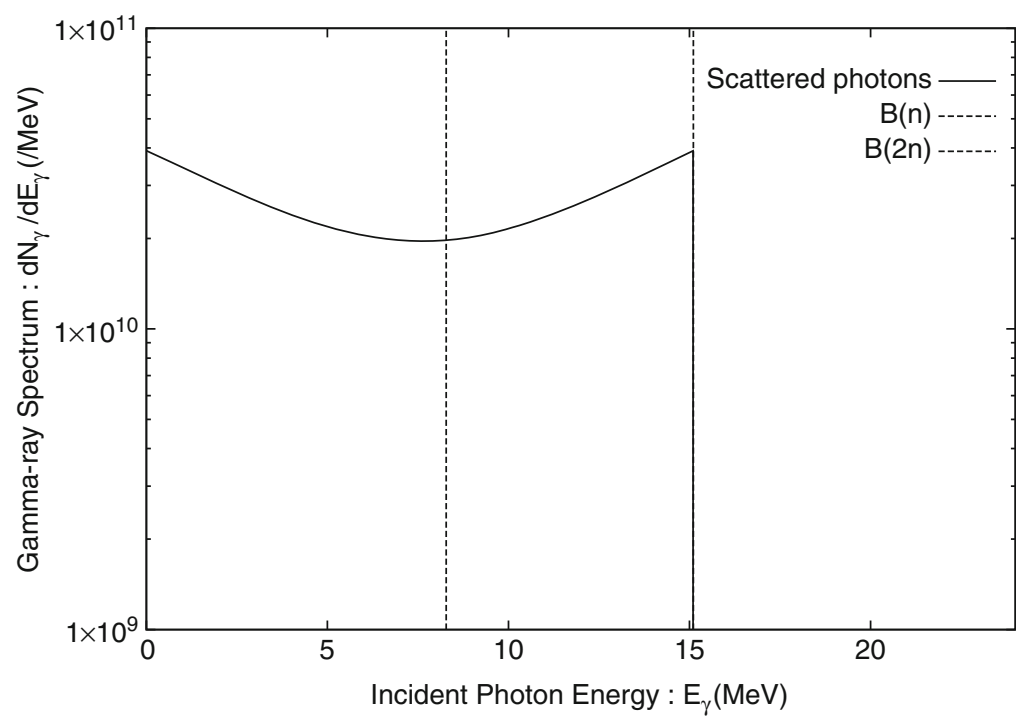

Fig. 1.3 Calculated gamma $(\gamma)$-ray spectrum (solid line) generated by laser Compton scattering. The maximum energy, $15 \mathrm{MeV}$, was chosen to be equal to the binding energy $B(2 \mathrm{n})$ of ${ }^{137} \mathrm{Cs}$. The binding energies of $B(\mathrm{n})$ and $B(2 \mathrm{n})$ for ${ }^{137} \mathrm{Cs}$ are indicated by dashed lines

To have a situation in which $(\gamma, \mathrm{n})$ reactions occur, the photon beam with energy at $B(\mathrm{n}) \leq E_{\gamma}<B(2 \mathrm{n})$ is desired. In case of the free electron laser, we may assume/ expect to get the total photon flux $N_{\gamma} \approx 2 \times 10^{12} / \mathrm{s} / 500 \mathrm{~mA}$ for $E_{e}=1.2 \mathrm{GeV}$ [1]. From Eq. (1.4), with $E_{e}=1.2 \mathrm{GeV}$ and $E_{L}=0.7 \mathrm{eV}$, we obtain the maximum photon energy of $E_{\gamma}=15 \mathrm{MeV}$ at $\theta=0$, which is equal to $B(2 \mathrm{n})$ for ${ }^{137} \mathrm{Cs}$. Figure 1.3 shows the calculated $\gamma$-ray spectrum generated by laser Compton scattering using Eq. (1.5), where the total photon flux with energy from 0 to $B(2 \mathrm{n})$ is $N_{\gamma} \approx 2 \times 10^{12} / \mathrm{s}$.

From Fig. 1.3, we can see that about half the total scattered photons are in $B(\mathrm{n}) \leq E_{\gamma}<B(2 \mathrm{n})$ and contribute to generate the $(\gamma, \mathrm{n})$ reactions for ${ }^{137} \mathrm{Cs}$. In contrast, for the Bremsstrahlung that is usually used to generate high-energy photons, the photon intensity decreases rapidly as the photon energy increases, and only a small part of the high-energy tail is available for $(\gamma, \mathrm{n})$ reactions [11].

\subsubsection{Setup of the Calculation for ${ }^{137} \mathrm{Cs}$}

When a target nucleus $\mathrm{X}$ is irradiated with a photon beam with energy $E_{\gamma}$, it forms a compound nucleus, which releases one neutron and becomes its isotope $X^{\prime}$. The reaction rate of $\mathrm{X}(\gamma, \mathrm{n}) \mathrm{X}^{\prime}$ at time $t$ is given by 


$$
r_{\mathrm{X} \rightarrow \mathrm{X}^{\prime}}(t)=\int_{B(\mathrm{n})}^{B(2 \mathrm{n})} \mathrm{d} E_{\gamma} \frac{\mathrm{d} N_{\gamma}}{\mathrm{d} E_{\gamma}} \sigma_{\mathrm{X} \rightarrow \mathrm{X}^{\prime}}\left(E_{\gamma}\right) n_{\mathrm{X}}(t) a,
$$

where $\sigma_{\mathrm{X} \rightarrow \mathrm{X}^{\prime}}\left(E_{\gamma}\right)$ is the reaction cross section of $\mathrm{X}(\gamma, \mathrm{n}) \mathrm{X}^{\prime}, \mathrm{n}_{\mathrm{X}}(t)$ is the number of target nucleus per unit area at time $t$, and $a$ is the attenuation factor of incident photons through a thick target. $\mathrm{d} N_{\gamma} / \mathrm{d} E_{\gamma}$ is expressed with Eq. (1.5) and $\sigma_{\mathrm{X}} \rightarrow \mathrm{X}^{\prime}\left(E_{\gamma}\right)$ is calculated from Eq. (1.2) using the TALYS code.

Figure 1.4 shows a calculation in which the photon beam is generated by the laser Compton scattering of $1.2 \mathrm{GeV}$ electrons and $0.7 \mathrm{eV}$ laser beams. We assume that the cylindrical target of ${ }^{137} \mathrm{Cs}$ of $1 \mathrm{~g}$ is irradiated with a photon beam with energy $B(\mathrm{n}) \leq E_{\gamma}<B(2 \mathrm{n})$ within a radius $r \approx 0.8 \mathrm{~mm}$ at $2 \mathrm{~m}$ from the interaction point. When a target of ${ }^{137} \mathrm{Cs}$ is irradiated with photons and is excited to GDR, the $(\gamma, \gamma),(\gamma, \mathrm{n})$ and $(\gamma, 2 \mathrm{n})$ reactions mainly occur. We consider ${ }^{137} \mathrm{Cs},{ }^{136} \mathrm{Cs},{ }^{135} \mathrm{Cs}$, and ${ }^{134} \mathrm{Cs}$ as the isotopes generated by the transmutation. The numbers of these isotopes are expressed as

$$
\begin{gathered}
n_{137}(t+\Delta t)=n_{137}(t) \mathrm{e}^{-\lambda_{137} \Delta t}-r_{137 \rightarrow 136}(t)-r_{137 \rightarrow 135}(t), \\
n_{136}(t+\Delta t)=n_{136}(t) \mathrm{e}^{-\lambda_{136} \Delta t}+r_{137 \rightarrow 136}(t)-r_{136 \rightarrow 135}(t)-r_{136 \rightarrow 134}(t), \\
n_{135}(t+\Delta t)=n_{135}(t) \mathrm{e}^{-\lambda_{135} \Delta t}+r_{137 \rightarrow 135}(t)+r_{136 \rightarrow 135}(t)-r_{135 \rightarrow 134}(t), \\
n_{134}(t+\Delta t)=n_{134}(t) \mathrm{e}^{-\lambda_{134} \Delta t}+r_{136 \rightarrow 134}(t)+r_{135 \rightarrow 134}(t) .
\end{gathered}
$$

One can calculate the number of each isotopes by solving these equations with the Runge-Kutta method.

\subsection{Results and Discussion}

\subsubsection{Nuclear Transmutation of ${ }^{137}$ Cs with Laser Compton Scattering}

Figure 1.5 shows the dependence of the reduction of $1 \mathrm{~g}{ }^{137} \mathrm{Cs}$ on the photon flux $N_{\gamma}=10^{12}, 10^{18}, 10^{19}, 10^{20} / \mathrm{s}$, which is calculated with this setup (Fig. 1.4). The number of ${ }^{137} \mathrm{Cs}$ is effectively reduced with photon flux over $10^{18} / \mathrm{s}$, that is, the number of ${ }^{137} \mathrm{Cs}$ is reduced by $10 \%$ for $24 \mathrm{~h}$ irradiation. Figure 1.6 shows the number of $\mathrm{Cs}$ isotopes when $1 \mathrm{~g}{ }^{137} \mathrm{Cs}$ is irradiated with photon flux $2 \times 10^{12} / \mathrm{s}$ with the same setup. From this figure, we can see that the reduction rate of ${ }^{137} \mathrm{Cs}$ by the transmutation, which is nearly equal to the generation rate of ${ }^{136} \mathrm{Cs}$, is two orders of magnitude smaller than the natural decay rate of $1 \mathrm{~g}{ }^{137} \mathrm{Cs}$. Thus, the transmutation of ${ }^{137} \mathrm{Cs}$ is not effective with photon flux $2 \times 10^{12} / \mathrm{s}$, which is maximum with present accelerator systems. 


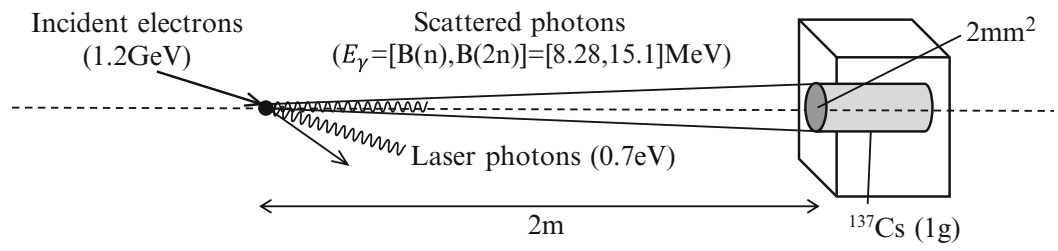

Fig. 1.4 Setup for calculation of transmutation of ${ }^{137} \mathrm{Cs}$

Fig. 1.5 Dependence of the reduction of $1 \mathrm{~g}{ }^{137} \mathrm{Cs}$ on photon flux $N_{\gamma}=10^{12}, 10^{18}, 10^{19}, 10^{20} / \mathrm{s}$

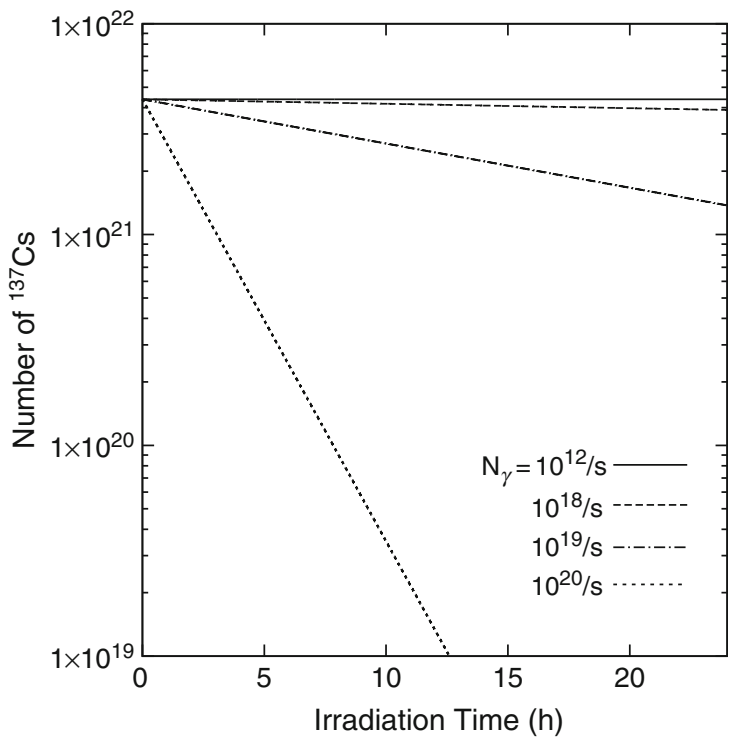

\subsubsection{Comparison with Other Nuclides}

Table 1.1 summarizes the generation rate of ${ }^{\mathrm{A}-1} \mathrm{X}$ from of $1 \mathrm{~g}$ of a fission product ${ }^{\mathrm{A}} \mathrm{X}$ with photon flux $2 \times 10^{12} / \mathrm{s}$ also for ${ }^{129} \mathrm{I},{ }^{135} \mathrm{Cs}$, and ${ }^{90} \mathrm{Sr}$ in addition to ${ }^{137} \mathrm{Cs}$. The reduction rate, which is nearly equal to the generation rate of ${ }^{\mathrm{A}-1} \mathrm{X}$, does not depend significantly on nuclides because the properties of GDR are smooth functions of the mass number. From this table, we can see that the reduction rate for the transmutation of ${ }^{137} \mathrm{Cs}$ can be similar to that of other medium nuclides. 
Fig. 1.6 Number of Cs isotopes when $1 \mathrm{~g}{ }^{137} \mathrm{Cs}$ is irradiated with photon flux $2 \times 10^{12} / \mathrm{s}$. Dotted line shows the number of ${ }^{137} \mathrm{Ba}$ that are generated by the natural decay of $1 \mathrm{~g}{ }^{137} \mathrm{Cs}$

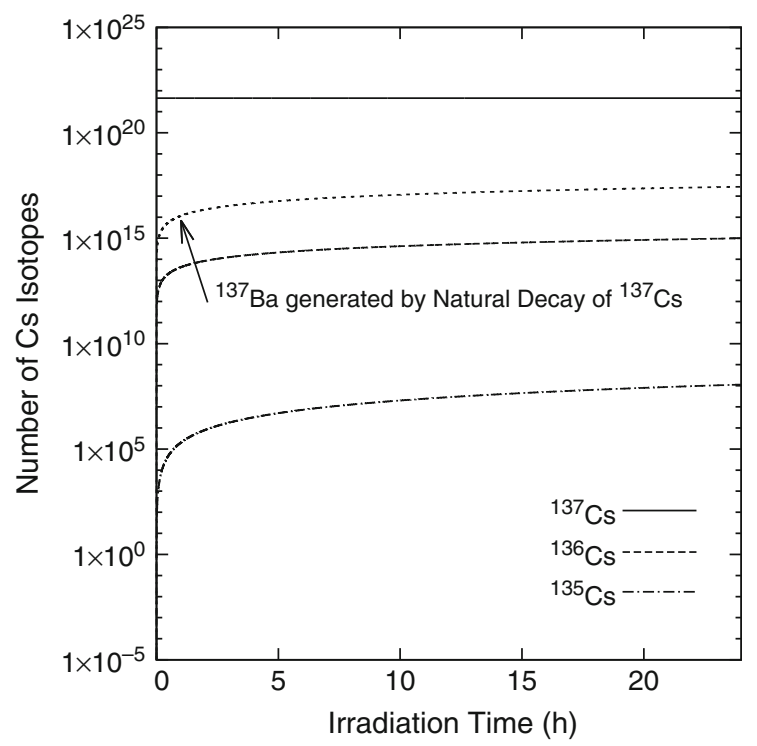

Table 1.1 Generation rate of ${ }^{\mathrm{A}-1} \mathrm{X}$ from $1 \mathrm{~g}$ of fission product ${ }^{\mathrm{A}} \mathrm{X}$ with photon flux $2 \times 10^{12} / \mathrm{s}$

\begin{tabular}{l|l|l|l|l}
\hline Target $\left({ }^{\mathrm{A}} \mathrm{X}\right)$ & {$[B(\mathrm{n}), B(2 \mathrm{n})](\mathrm{MeV})$} & $E_{\mathrm{GDR}}(\mathrm{MeV})$ & $\sigma_{\mathrm{GDR}}^{\text {tot }}(\mathrm{b} \cdot \mathrm{MeV})$ & $N\left({ }^{\mathrm{A}-1} \mathrm{X}\right)(/ \mathrm{s})$ \\
\hline${ }^{129} \mathrm{I}$ & {$[8.83,15.7]$} & 15.3 & 2.25 & $1.24 \times 10^{10}$ \\
\hline${ }^{135} \mathrm{Cs}$ & {$[8.76,15.7]$} & 15.2 & 2.31 & $1.31 \times 10^{10}$ \\
${ }^{137} \mathrm{Cs}$ & {$[8.28,15.1]$} & 15.1 & 2.37 & $1.19 \times 10^{10}$ \\
${ }^{90} \mathrm{Sr}$ & {$[7.81,14.2]$} & 16.7 & 1.58 & $4.71 \times 10^{9}$ \\
\hline
\end{tabular}

\subsection{Conclusion}

In this work, the effectiveness of transmutation with laser Compton scattering for reducing fission products was quantitatively investigated. The transmutation of ${ }^{137} \mathrm{Cs}$ is effective with photon flux greater than $10^{18} / \mathrm{s}$, which results in $10 \%$ reduction for $24 \mathrm{~h}$ irradiation. However, transmutation with photon flux $2 \times 10^{12} / \mathrm{s}$, which is achievable with present maximum accelerator systems, is not effective, and the reduction rate is approximately two orders of magnitude less than the natural decay rate.

Nuclear transmutation with laser Compton scattering can transmute selectively a medium mass nuclide ${ }^{\mathrm{A}} \mathrm{X}$ into ${ }^{\mathrm{A}-1} \mathrm{X}$, and its reduction rate is independent of isotopes. Because the transmutation with laser Compton scattering can almost exclusively generate desired nuclides, this method will be useful for the generation of isotopes for medicine [1]. 
Open Access This chapter is distributed under the terms of the Creative Commons Attribution Noncommercial License, which permits any noncommercial use, distribution, and reproduction in any medium, provided the original author(s) and source are credited.

\section{References}

1. Ejiri H, Shima T, Miyamoto S, Horikawa K, Kitagawa Y, Asano Y, Date S, Ohashi Y (2011) J Phys Soc Jpn 80:094202

2. Li D, Imasaki K, Horikawa K, Miyamoto S, Amano S, Mochizuki T (2009) J Nucl Sci Technol 46:8

3. Bohr A, Mottelson B $(1969,1975)$ Nuclear structure, vols I and II. Benjamin, New York

4. Capote R, Herman M, Oblozinsky P, Young PG, Goriely S, Belgya T, Ignatyuk AV, Koning AJ, Hilaire S, Plujko V, Avrigeanu M, Bersillon O, Chadwick MB, Fukahori T, Kailas S, Kopecky J, Maslov VM, Reffo G, Sin M, Soukhovitskii E, Talou P, Yinlu H, Zhigang G (2009) Nucl Data Sheets 110:3107

5. Maeda K, Shibata T, Ejiri H (1983) Phys Rev C 28:635

6. Koning AJ, Hilaire S, Duijvestijn MC (2008) TALYS-1.4. In: Bersillon O, Gunsing F, Bauge E, Jacqmin R, Leray S (eds) Proceedings of the International Conference on Nuclear Data for Science and Technology, vol 211, Nice, April 22-27 2007. EDP Sciences, . See also http://www.talys.eu/documentation/

7. Koning AJ, Delaroche JP (2003) Nucl Phys A 713:231

8. Brink DM (1967) Nucl Phys 4:215

9. Axel P (1962) Phys Rev 126:671

10. Gilbert A, Cameron AGW (1965) Can J Phys 43:1446

11. Bunatiana GG, Nikolenko VG, Popov AB (2010) arXiv 1012.5002vl [nucl-exp, Dec 2010]. http://arxiv.org/pdf/1012.5002.pdf 